\title{
A New CAD System for the Evaluation of Kidney Diseases Using DCE-MRI
}

\author{
Ayman El-Baz ${ }^{1}$, Rachid Fahmi ${ }^{1}$, Seniha Yuksel ${ }^{1}$, Aly A. Farag ${ }^{1}$, \\ William Miller ${ }^{1}$, Mohamed A. El-Ghar ${ }^{2}$, and Tarek Eldiasty ${ }^{2}$ \\ ${ }^{1}$ Computer Vision and Image Processing Laboratory, \\ University of Louisville, Louisville, KY 40292 \\ \{elbaz, rachidf, esen, farag\}@cvip.uofl.edu \\ http://www.cvip.louisville.edu . \\ ${ }^{2}$ Urology and Nephrology Department, \\ University of Mansoura, Mansoura, Egypt
}

\begin{abstract}
Acute rejection is the most common reason of graft failure after kidney transplantation, and early detection is crucial to survive the transplanted kidney function. In this paper, we introduce a new approach for the automatic classification of normal and acute rejection transplants from Dynamic Contrast Enhanced Magnetic Resonance Imaging (DCEMRI). The proposed algorithm consists of three main steps; the first step isolates the kidney from the surrounding anatomical structures by evolving a deformable model based on two density functions; the first function describes the distribution of the gray level inside and outside the kidney region and the second function describes the prior shape of the kidney. In the second step, a new nonrigid registration approach is employed to account for the motion of the kidney due to patient breathing. To validate our registration approach, we use a simulation of deformations based on biomechanical modelling of the kidney tissue using the finite element method (F.E.M.). Finally, the perfusion curves that show the transportation of the contrast agent into the tissue are obtained from the cortex and used in the classification of normal and acute rejection transplants. Applications of the proposed approach yield promising results that would, in the near future, replace the use of current technologies such as nuclear imaging and ultrasonography, which are not specific enough to determine the type of kidney dysfunction.
\end{abstract}

\section{Introduction}

In the United States, approximately 12000 renal transplants are performed annually [1], and considering the limited supply of donor organs, every effort is made to salvage the transplanted kidney [2]. Currently, the diagnosis of rejection is done via biopsy which has the downside effect of subjecting the patients to risks such as bleeding and infections. Moreover, the relatively small needle biopsies may lead to over or underestimation of the extent of inflammation in the entire graft [3]. Therefore, a noninvasive and repeatable technique is not only helpful but also needed in the diagnosis of acute renal rejection. In DCE-MRI, a 
contrast agent called Gd-DTPA is injected into the bloodstream, and as it perfuses into the organ, the kidneys are imaged rapidly and repeatedly. During the perfusion, Gd-DTPA causes a change in the relaxation times of the tissue and creates a contrast change in the images. As a result, the patterns of the contrast change gives functional information, while MRI provides good anatomical information which helps in distinguishing the diseases that affect different parts of the kidneys. However, even with an imaging technique like DCE-MRI, there are several problems such as, (i) the spatial resolution of the dynamic MR images is low due to fast scanning, (ii) the images suffer from the motion induced by the patient breathing which necessitates advanced registration techniques, (iii) the intensity of the kidney changes non-uniformly as the contrast agent perfuses into the cortex which complicates the segmentation procedures.

To the best of our knowledge, there has been limited work on the dynamic MRI to overcome the problems of registration and segmentation. For the registration problem, Gerig et al. 4 proposed, using Hough transform, to register the edges in an image to the edges of a mask and Giele et al. [5] introduced a phase difference movement detection method to correct for kidney displacements. Both of these studies required building a mask manually by drawing the kidney contour on a 2D DCE-MRI image, followed by the registration of the time frames to this mask.

Most of these efforts used healthy transplants in the image analysis, and edge detection algorithms were sufficient. However; in the case of acute rejection patients, the uptake of the contrast agent is decreased, so edge detection fails in giving connected contours. For this reason, we consider the combined usage of gray level and prior shape information to give better results.

\section{Methods}

In this paper we introduce a novel and automated technique (i) to segment the kidney and (ii) to correct for the motion artifacts caused by breathing and patient motion. The details of the techniques are given below.

\subsection{Segmentation}

The first step of the proposed approach is to extract the kidney tissues from DCE-MRI's as shown in Fig. 1. Segmentation algorithms are based on using the deformable model guided by a stochastic force which represents the intensity and shape prior of the kidney. Details of the algorithm are presented in [6].

\subsection{Model for the Local Deformation}

In DCE-MRI sequences, the registration problem arises because of the patient and breathing movements. To solve this problem, we propose a new approach to handle the kidney motion. The proposed approach is based on deforming the segmented kidney over evolving closed equispaced contours (i.e. iso-contours) to closely match the prototype. The evolution of the iso-contours is guided by 


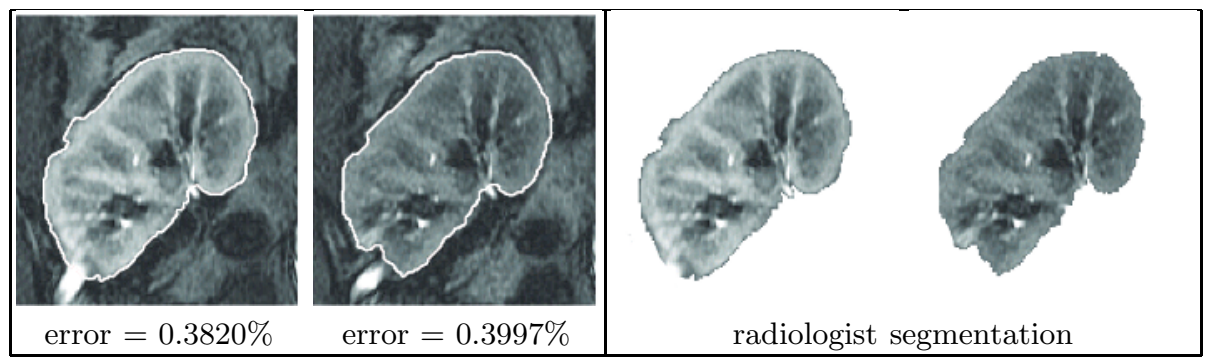

Fig. 1. Segmentation results using the approach proposed in [6] with the errors w.r.t the radiologist segmentation

an exponential speed function in the directions minimizing distances between corresponding pixel pairs on the iso-contours of the objects to be registered. The normalized cross-correlation is used as an image similarity measure which is insensitive to intensity changes (e.g. due to tissue motion in medical imagery and the contrast agent). Unlike free-form deformation approaches based on B-spline, our technique is less expensive computationally.

The first step of the proposed registration approach is to use the fast marching level set method [7] to generate the distance map inside the kidney regions as shown in Fig. 2(a)-(b). The second step is to use this distance map to generate equal space separated contours (iso-contours) as shown in Fig. 2(c)-(d). Note that the number of iso-contours depends on the accuracy and the speed required by the user. The third step of the proposed approach is to use normalized cross correlation to find the correspondence between the iso-contours. Since we start with aligned images, we limit our searching space to a small window (e.g. $10 \times 10)$ to improve the speed of the proposed approach. The final step is the evolution of the iso-contours; here, our goal is to deform the iso-contours in the first image (target image) to match the iso-contours in the second image (source image). Before discussing the details of the evolution algorithm, let's define the following:

$-\phi_{n_{\text {iso }}}^{A}(., \nu)$ are the iso-contours in the target image $(A)$, where $n_{\text {iso }}=1, \ldots, N_{\text {iso }}$ is the index of the iso-contours, and $\nu$ the iteration step,

$-\phi_{m_{\text {iso }}}^{B}($.$) are the iso-contours in the source image (B)$, where $m_{\text {iso }}=1, \ldots, M_{\text {iso }}$ is the index of iso-contours,

- $S\left(h, \gamma_{h}\right)$ denotes the Euclidean distance between a iso-contour point $h$ on image $A$ and its corresponding iso-contour point $\gamma_{h}$ on image $B$. Note that $\gamma_{h}$ is searched for within a local window centered at $h^{\prime} s$ position in image B. Note also that $\gamma_{h}$ may be the same for different $h^{\prime} s$,

- $S_{n_{\text {iso }}, n_{\text {iso }}-1}^{A}(h)$ is the Euclidian distance between $\phi_{n_{\text {iso }}}^{A}(h, \nu)$ and $\phi_{n_{\text {iso }}-1}^{A}(h, \nu)$ at each iteration $\nu$,

$-\mathbf{V}($.$) is the propagation speed function.$

The most important step in the model propagation is the selection of the propagation speed function $\mathbf{V}($.$) . This selection must satisfy the following con-$ ditions: 
1. $\mathbf{V}(h)=0$ if $S\left(h, \gamma_{h}\right)=0$,

2. $\mathbf{V}(h) \leq \min \left(S\left(h, \gamma_{h}\right), S_{n_{\text {iso }}, n_{\text {iso }}-1}^{A}(h), S_{n_{\text {iso }}, n_{\text {iso }}+1}^{A}(h)\right)$ if $S\left(h, \gamma_{h}\right)>0$; is the smoothness constraint, which prevents the current point from cross passing the closest neighbor contour as shown in Fig. 2(e).

The following speed function satisfies the above conditions:

$$
\mathbf{V}(h)=e^{\beta(h) \cdot S\left(h, \gamma_{h}\right)}-1,
$$

where $\beta(h)$ is the propagation constant with the upper bound

$$
\beta(h) \preceq \frac{\ln \left(\min \left(S\left(h, \gamma_{h}\right), S_{n_{\text {iso }}, n_{\text {iso }}-1}^{A}(h), S_{n_{\text {iso }}, n_{\text {iso }}+1}^{A}(h)\right)+1\right)}{S\left(h, \gamma_{h}\right)} .
$$

Based on this speed function, we can deform the iso-contours using the following equation as shown in Fig. 2(f):

$$
\phi^{A}(h, \nu+1)=\frac{\mathbf{V}(h)}{S\left(h, \gamma_{h}\right)} \phi_{m_{i s o}}^{B}\left(\gamma_{h}\right)+\frac{S\left(h, \gamma_{h}\right)-\mathbf{V}(h)}{S\left(h, \gamma_{h}\right)} \phi_{n_{i s o}}^{A}(h, \nu)
$$

where $h=1, \ldots, \mathcal{H}$ denotes a point on the iso-contours on image $A$, and $\gamma_{h}$ its corresponding point on image $B$.

To show the quality of the proposed approach, we fused the two kidney images by a checkerboard visualization in Fig. 3. It is clear from Fig. 3. (b) that the connectivity between the two images at the edges and inside the kidney region are improved after applying the proposed deformation model.

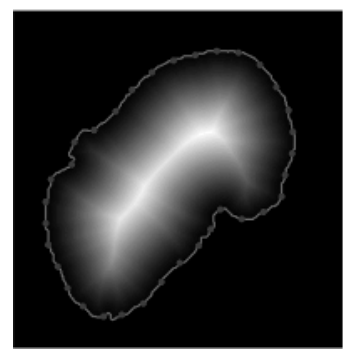

(a)

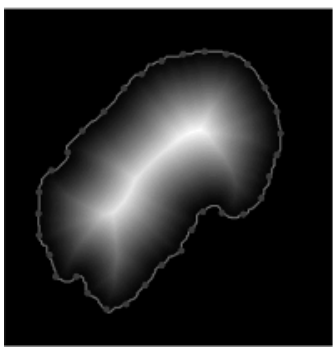

(b)

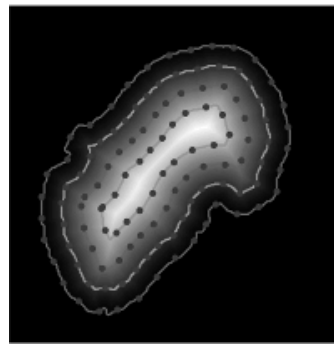

(c)

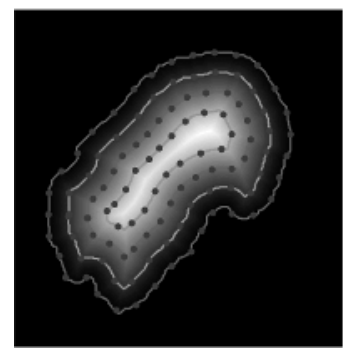

(d)

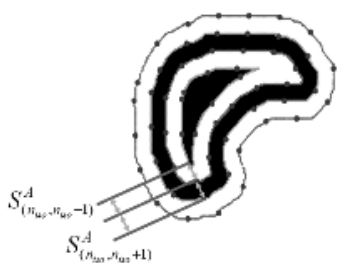

(e)

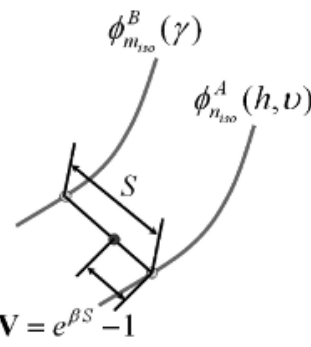

Fig. 2. The distance map of two kidneys (a, b) and the iso-contours (c, d), a model constrains (e), and a model evolution (f) 

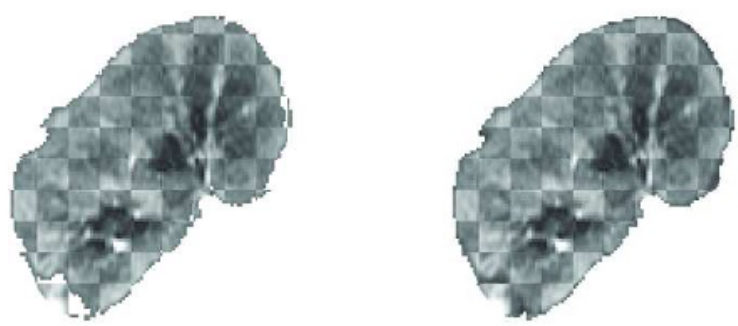

(a)

Fig. 3. Checkerboard image to show the quality of the approach, before non-rigid registration (a), and after non-rigid registration (b)

\subsection{Validation of Our Registration Approach Using the F.E.M.}

In this section we propose to validate our deformable registration approach using the finite element (F.E.) method. Given a 2D image of the kidney, we simulate a deformation using a biomechanical modelling of the kidney tissue. The pair of images (deformed and non-deformed ones) is used to test our algorithm. The Abaqus/CAE (Ver. 6.5) 1 environment was used to generate a cubic spline fit

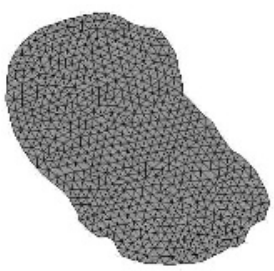

(a)

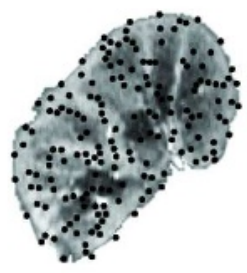

(e)

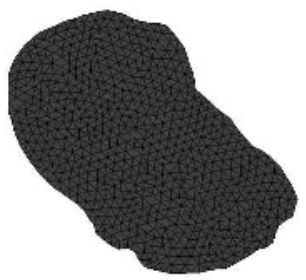

(b)

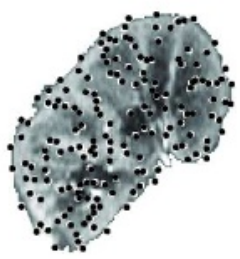

(f)

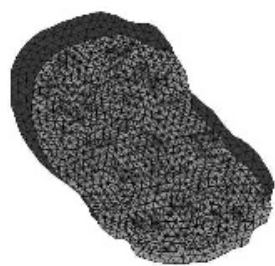

(c)

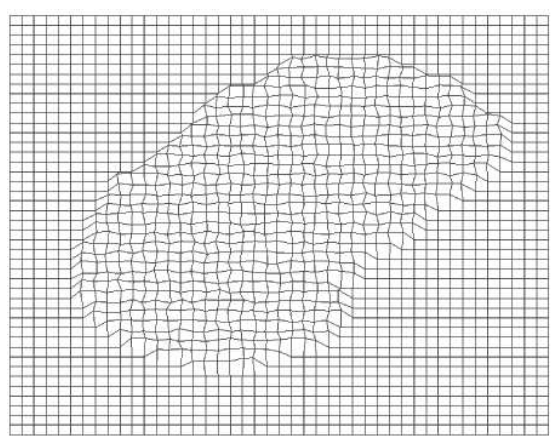

(g)

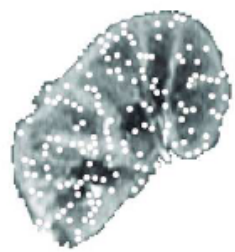

(d)

Fig. 4. Validation of non-rigid registration. F.E mesh (a) before and (b) after deformation. (c) Meshes (a) \& (b) overlayed. (d)\&(e) Sample of the selected correspondence pairs determined from F. E meshes before \& after deformation. (f) Results of the proposed non-rigid registration approach. (g) The displacement field.

\footnotetext{
${ }^{1}$ www.abaqus.com
} 
to the points representing the outer contour of the kidney object and then a 2D F.E. model was built from it. Figure 4(a), (b) and (c) show the 2D mesh before and after deformation, and the overlay of these two meshes, respectively. For the sake of generating a deformed shape only, we assumed the kidney tissue to be isotropic and homogeneous elastic material with a Young Modulus $E=2500 \mathrm{~Pa}$ and a Poisson Ratio $\nu=0.4$. Note that this model does not reflect the results of any rheological experiments conducted on the kidney tissue. A uniformly distributed pressure $P=100 N$ was applied normal to the boundary of the kidney. The points on this boundary are allowed to move freely in the $x$ and $y$ directions, but are constrained to rotate around the $z$-axis. The mesh consists of 1253 3-node linear plane stress angular elements. The average displacement of the induced deformation is $4.75 \mathrm{~mm}$, the minimum is $1.19 \mathrm{~mm}$, and the maximum is $6.9 \mathrm{~mm}$. The accuracy of the registration method is assessed by registering the simulated deformed image to the original one and comparing the recovered point displacements with the bio-mechanically simulated ones. The average registration error is about $1.54 \mathrm{~mm}$, with a maximum of $2.7458 \mathrm{~mm}$, a minimum of $0.0221 \mathrm{~mm}$, and a standard deviation of 0.5517 . This proves the accuracy of our non-rigid registration technique used in this work.

\subsection{Cortex Segmentation}

Strake et.al. 8 had shown that the most important signal characteristics come from the cortex of the kidney in the acute rejection problem. Therefore, the final step of our approach is to segment the cortex from the segmented kidney. To achieve this task, we use the same segmentation approach shown in [6] but based only on the intensity. At this step, since all the kidneys are aligned together, we select seed points from the medulla regions and evolve the deformable model based only on intensity. After we extract the medullary regions, the rest is cortex, which is used as a mask and propagated over the whole sequence to plot the average cortex intensity. In Fig. [5, we show the cortex segmentation results. In Fig. [5(a), we manually initialize several deformable models inside medulla regions, and we let the deformable model to evolve in these regions with gray

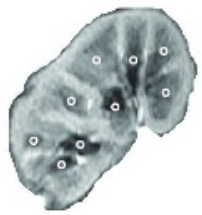

(a)

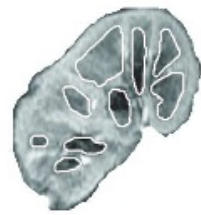

(b)

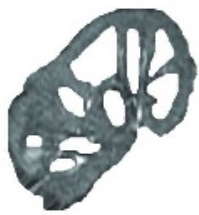

(c)

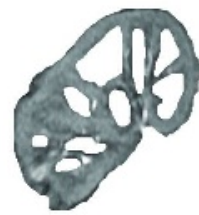

(d)

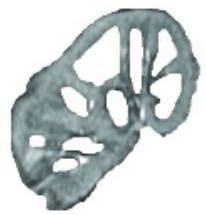

(e)

Fig. 5. The segmentation of the cortex from the kidney images. Several medullary seeds are initialized (a), and the deformable model grows from these seed point (b). After the medulla is extracted from the kidney, the cortex is propagated over the whole sequence of images as shown in (c)-(e). 


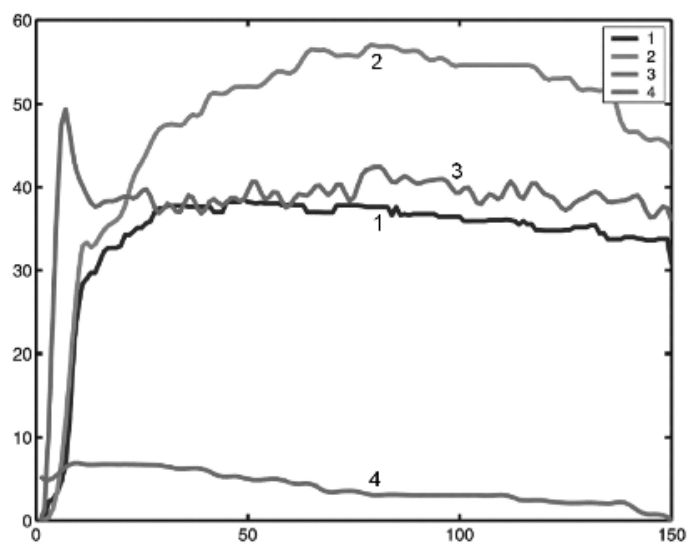

Fig. 6. Normalized cortex signals from 4 subjects w.r.t. scan number. Subjects 1 and 2 are acute rejection, subject 3 is normal and subject 4 is chronic glomerulopathy proved by biopsy.

level information as shown in Fig. 5(b). The cortex mask is applied to the rest of the sequence as shown in Fig 5 (c)-(e).

\section{Results and Conclusion}

The ultimate goal of the proposed algorithms is to successfully construct a renogram (mean intensity signal curves) from the DCE-MRI sequences, showing the behavior of the kidney as the contrast agent perfuses into the transplant. In acute rejection patients, the DCE-MRI images show a delayed perfusion pattern and a reduced cortical enhancement. We tested the above algorithms on thirty patients, four of which are shown in Figure 6. The normal patient shows the expected abrupt increase to the higher signal intensities and the valley with a small slope. The acute rejection patients show a delay in reaching their peak signal intensities. From these observations, we have been able to conclude that the relative peak signal intensity, time to peak signal intensity, the slope between the peak and the first minimum, and the slope between the peak and the signal measured from the last image in the sequence are the major four features in the renograms of the segmented kidney for classification.

To distinguish between normal and acute rejection, we use Bayesian supervised classifier learning statistical characteristics from a training set for the normal and acute rejection. The density estimation required in the Bayes classifier is performed for each feature by using a linear combination of Gaussians (LCG) with positive and negative components. The parameters of the LCG components are estimated using a modified EM algorithm [9]. In our approach, we used 50\% of the data for the training and the other $50 \%$ for testing. For testing data, the Bayes classifier succeeds to classify 13 out of 15 correctly $(86.67 \%)$. For the training data, the Bayes classifier classifies all of them correctly, so the overall accuracy of the proposed approach is $93.3 \%$. 
In this paper, we presented a framework for the detection of acute renal rejection from DCE-MRI which includes segmentation of the kidneys from the abdomen images, non-rigid registration and Bayes classification. Our future work will include testing on more patients; the results of the proposed framework are promising and might replace the current nuclear imaging tests or the invasive biopsy techniques.

\section{References}

1. U.S. Department of Health and Human Services. Annual report of the U.S. scientific registry of transplant recipients and the organ procurement and transplantation network: transplant data: 1990-1999. Bureau of Health Resources Department, Richmond, VA; 2000.

2. M. Neimatallah, Q. Dong, S. Schoenberg, K. Cho, and M. Prince, "Magnetic resonance imaging in renal transplantation," Journal of Magnetic Resonance Imaging, vol. 10(3), pp. 357-368, Sep 1999.

3. D. Yang, Q. Ye, M. Williams, Y. Sun, T. C. C. Hu, D. S. Williams, J. M. F. Moura, and C. Ho., "USPIO-Enhanced Dynamic MRI: Evaluation of Normal and Transplanted Rat Kidneys," Magnetic Resonance in Medicine, vol. 46, 1152-1163, 2001.

4. G. Gerig, R. Kikinis, W. Kuoni, G.K. van Schulthess, and O. Kubler, "Semiautomated ROI analysis in dynamic MRI studies: Part I: image analysis tools for automatic correction of organ displacements," IEEE Transactions Image Processing, vol. 11:(2),pp. 221-232, 1992.

5. E. Giele, "Computer methods for semi-automatic MR renogram determination," Ph.D. dissertation, Department of Electrical Engineering, Eindhoven University of Technology, Eindhoven, 2002.

6. A. El-Baz, S. E. Yuksel, A. A. Farag, H. Shi, T. El-Diasty, and M. Ghoneim, "2D and 3D Shape Based Segmentation Using Deformable Model," Proc. of International Conference on Medical Image Computing and Computer-Assisted Intervention, MICCAI-2005, Palm Springs, California, USA, October 26-29, 2005.

7. J. A. Sethian, "Fast marching level set method for monotonically advancing fronts," Proceedings of the National Academy of Sciences, USA, vol. 93, pp. 1591-1595, Feb. 1996.

8. L. Strake, L. Kool, L. Paul. A. Tegzess, J. Weening, J. Hermans, J. Doornbos, R. Bluemm, and J. Bloem, "Magnetic resonance imaging of renal transplants: its value in the differentiation of acute rejection and cyclosporin A nephrotoxicity," Journal of Clinical Radiology, vol. 39(3), pp. 220-228, May, 1988.

9. A. A. Farag, A. El-Baz, and G. Gimel'farb, "Precise Segmentation of Multi-modal Images," IEEE Transactions on Image Processing, vol. 15, no. 4, pp. 952-968, April 2006 . 